\title{
¿Progressive Pulse Compression: A Novel Technique for Blind Range Recovery for Solid-State Radars
}

\author{
Cesar M. Salazar Aquino, ${ }^{\mathrm{a}}$ Boonleng Cheong, ${ }^{\mathrm{b}}$ and Robert D. Palmer ${ }^{\mathrm{c}}$

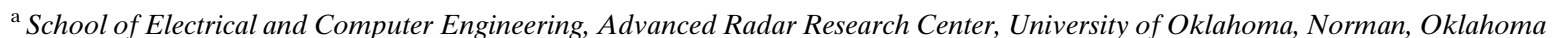 \\ ${ }^{\mathrm{b}}$ Advanced Radar Research Center, University of Oklahoma, Norman, Oklahoma \\ ${ }^{\mathrm{c}}$ School of Meteorology, Advanced Radar Research Center, University of Oklahoma, Norman, Oklahoma
}

(Manuscript received 11 October 2020, in final form 24 June 2021)

\begin{abstract}
In this paper, a novel technique is proposed to mitigate the so-called blind range on radars that use pulse compression. It is well known that the blind range is caused by the strong leak through into the receiver during the transmission cycle. The proposed technique is called progressive pulse compression (PPC) and is based on partial decoding. PPC uses a portion of the uncontaminated received signal in conjunction with pulse compression to estimate the echoes from the incomplete signal. The technique does not require the use of a fill pulse or any hardware modifications. PPC can be divided into three steps. The first step is to discard all the received signals during the transmit cycle and apply a smooth taper for continuous transition from zero to one. The second step is to perform the pulse compression using matched filter. The combination of these two steps is equivalent to performing pulse compression using a progressively changing template to partially extract the uncontaminated received signal for compression. The third step is to compensate for the progressively changing template so that proper reflectivity values can be recovered. This technique has been tested on the PX-1000 and will be implemented on PX-10k in the near future. These two radars are designed and operated by the Advanced Radar Research Center at the University of Oklahoma and are both X-band software-defined solid-state systems. The results presented in this paper are collected using the PX-1000 radar.
\end{abstract}

KEYWORDS: Algorithms; Radars/Radar observations; Weather radar signal processing; Software

\section{Introduction}

Radar systems are one important tool used in many applications including the study of severe weather, defense, and autonomous driving (Doviak and Zrnić 2006b; Balajti et al. 2012; Hysell 2018a). Due to its portability and low cost, an increasingly popular radar architecture used in modern weather applications is one that is based on a solid-state transmitter (Wada et al. 2009). Researchers at the Advanced Radar Research Center (ARRC) of the University of Oklahoma have developed two radar systems using this solid-state technology.

Solid-state radars typically have a lightweight transmitter, which reduces the size and cost of the system. However, solidstate transmitters generally have lower peak power, which causes the loss of radar sensitivity. To compensate for this limitation, a long pulse is typically used. On the other hand, since the range resolution is inversely proportional to the pulse width, it suffers the loss of range resolution. This is generally overcome by using a technique called pulse compression, which requires the modulation of the pulse, either in frequency, which can be a linear frequency modulation (LFM) or a nonlinear frequency modulation (NLFM) (Keeler and Hwang 1995; De Witte and Griffiths 2004; Kurdzo et al. 2014),

\footnotetext{
D Denotes content that is immediately available upon publication as open access.

Corresponding author: Cesar M. Salazar Aquino, cesar.salazar@ ou.edu
}

phase, etc. The range resolution of a pulse compression system is proportional to the bandwidth of the waveform (Keeler and Hwang 1995; Mudukutore et al. 1998; Skolnik 2001b; Doviak and Zrnić 2006a). The success of a solid-state radar hinges upon the performance of its pulse compression waveforms. Moreover, the popularity of solid-state radars has driven interest over pulse compression in the weather radar field, making this solution even more useful.

In the atmospheric science community, pulse compression has been studied and applied to weather radars since the 1990s (e.g., Keeler and Hwang 1995; Mudukutore et al. 1998; O'Hora and Bech 2007; George et al. 2008; Pang et al. 2015; Torres et al. 2017; Hysell 2018b). For convenience, in this work, the existing pulse compression process will be called legacy pulse compression (LPC).

On solid-state radar systems, the use of a long transmitted waveform introduces the so-called blind range, which is a welldocumented problem through the literature in weather radars (O'Hora and Bech 2007; George et al. 2008, 2010; Bharadwaj and Chandrasekar 2012; Cheong et al. 2013; Nguyen and Chandrasekar 2014; Kurdzo et al. 2014). In general, blind range is a portion of the received data that is obscured due to leakage (i.e., leak through) from the long transmit waveform to the receiver. The obscured portion, or blind range $R_{b}$, can be several kilometers long, and it is directly proportional to the pulse width, as described by Eq. (1):

$$
R_{b}=\frac{c \tau}{2}
$$

where $c$ is the speed of light ( $\mathrm{m} \mathrm{s}^{-1}$ ) and $\tau$ is the pulse width (s). It is clear that a longer pulse result in a longer blind range. 
For example, the PX-1000 transmits a waveform with a pulse width of $69 \mu$ s (time-frequency multiplex of a $67-\mu$ s long pulse and a $2-\mu$ s fill pulse), as described in Cheong et al. (2013), and has a blind range of approximately $10 \mathrm{~km}$.

When the radar is transmitting, it is usually not possible to receive useful data. There are some exceptions in which the transmitted signal is intentionally sampled (through a separate channel) to measure both peak power and instantaneous phase at the peak point, the so-called burst pulse. Transmitter and receiver chains in radars are usually separated and isolated within the hardware using a switch or circulator. However, hardware devices do not provide perfect isolation, and a highpower copy of the transmitted waveform gets leaked (coupled) into the receiver causing what is termed a leak through. This leak through causes the blind range as the leak through overpowers the received signals from the atmosphere and, thus, the radar is overwhelmed by the leaked energy, effectively saturating the receiver. Therefore, weather signals inside this region are obscured. As explained before, blind range is a problem for solid-state radars because it can span up to tens of kilometers, limiting the quantity of useful data that the radar can provide.

Various solutions have been investigated to recover the data from the blind range. Proposed by Bharadwaj and Chandrasekar (2012), one such option is the use of multiple frequency bands and pulse lengths in which the pulses are transmitted successively. Another method uses a second pulse that is time-frequency multiplexed with the long pulse, documented in Cheong et al. (2013). Finally, the use of multiple pulses separated in frequency and time has also been suggested, presented by George et al. (2010). The downside of these methods is the additional bandwidth to accommodate the extra pulses. Also, the extra pulses used in these methods are shorter, resulting in a lower integrated power and an abrupt change in radar sensitivity between the blind range and visible range.

The progressive pulse compression (PPC) technique proposed in this paper recovers the blind range and requires no fill pulses. It uses the residual signals that are not contaminated by the leak through. This technique first eliminates the leak through and replaces it with zeroes; this does not eliminate the entirety of the echoes from obscured targets inside the blind range but only a portion of them. Since the uncompressed pulse is so long, even when a portion of it is covered by the leak through, a tail portion extends outside of this blind range and the return is uncontaminated. This tail portion is used to estimate the target signals within the blind range. Of course, because of the partial return, a proper calibration must be applied.

The main advantage of the PPC is that no additional bandwidth is required. In addition, compared to the previously proposed methods, since a single long pulse is used, the sensitivity is higher inside the blind range. The PPC is, in general, less expensive, in both bandwidth and computational requirements, to implement.

The rest of this paper is structured in two main sections. Section 2 describes the methodology. Section 3 details how capable this technique is in uncovering obscured data inside the blind range. Results from both simulated and actual data gathered from the PX-1000 radar system will be presented.

\section{Methodology}

\section{a. Algorithm description}

The PPC technique is intended for blind range mitigation for solid-state radars but is applicable to other types of radars that transmit FM pulses for pulse compression. Partial decoding and pulse compression methods are employed where the data from different temporal samples are coherently integrated into a single sample using pulse compression. Even if some data are lost or altered due to the leak through, pulse compression allows recovery of the blind range using remaining data that have not been altered.

The algorithm can be divided into three steps. First it is necessary to eliminate the leak through from the transmission into the receiver and then partially decode and compress the residual tail of the received signal. Finally, it is calibrated to correctly estimate the signal power or target reflectivity. This is necessary since only a portion of the received signal is used for compression. A flowchart of the PPC technique is shown in Fig. 1.

Partial decoding has been attempted using noiselike waveforms, studied in Pralon et al. (2012), but only simulated results were reported. A drawback of that technique is that it does not account for the leak through, a problem common in radars due to hardware limitations. Also, the work does not offer a calibration solution to deal with the gain problem caused on the compressed received signal. This paper will present experimental results from a real radar system, that is, the PX-1000.

\section{1) LEAK THROUGH ELIMINATION AND PARTIAL DECODING}

From Fig. 2, it can be observed that the received echoes, which are compressed to obtain point targets, have the same width as the transmit signal. If a target is located inside the blind range, regular implementations render it unusable because the leak through from transmission will mask the desired signal. This can be seen in the conventional method column. In there, signals received by the radar system are added, producing a composite signal, which includes the leak through and the echoes from the targets; see the yellow line in the conventional method. However, saturation is part of the equation, and it caps the maximum power that the radar can receive (saturation level); see the pink line in the conventional method. As a consequence, the leak through saturates the received signal inside the blind range, and the contribution from targets there is now obscured.

Nonetheless, since the signal received from each target is long, there is a portion free from this contamination, and not affected by the saturation (see the pink line in the new method column in Fig. 2). Then, in reality, if the target is located inside the blind range, the transmission leak through only masks a portion of the return echo, leaving a tail portion free from the leak-through contamination. Data from the tail portion can be used to recover the obscured but desired target. 


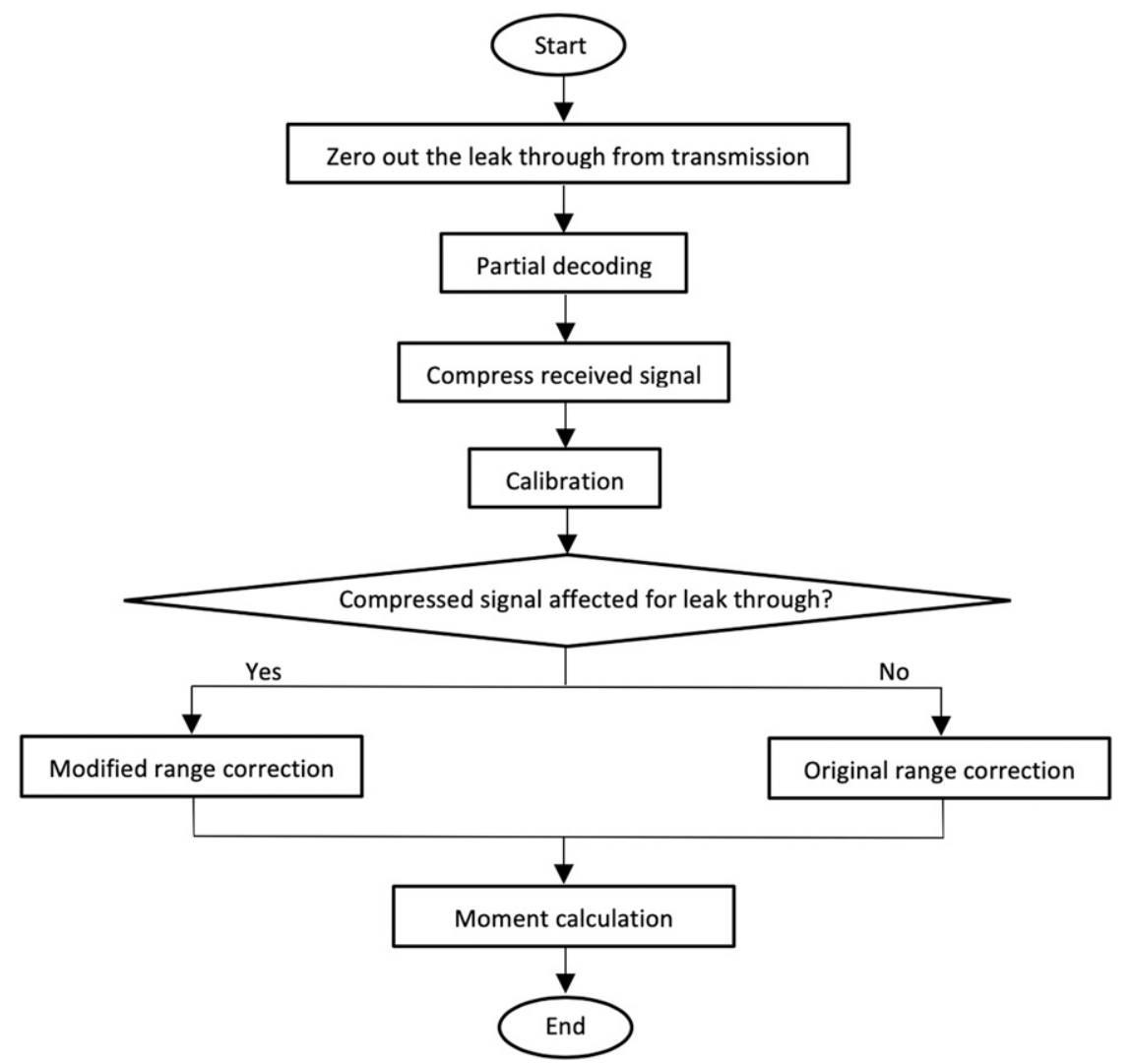

FIG. 1. Flowchart of the PPC technique. Key differences from the PPC include the use of a window function to zero out the leak through from transmission, the partial decoding, and the use of a new calibration factor to estimate the reflectivity inside the blind range.

The principle behind partial decoding is using uncontaminated data from the tail portion of the uncompressed received signal to predict the target. To achieve this goal, only tail portion data are used for compression and are processed ignoring the data from the other portion of the signal; thus, the term partial decoding was chosen. For example, assuming a 10-km pulse, the blind range is also approximately $10 \mathrm{~km}$. If a target is located at $3 \mathrm{~km}$, the signal return from the target is observed at $3 \mathrm{~km}$. However, since the pulse is $10 \mathrm{~km}$ long, the tail of the return is observed at $13 \mathrm{~km}$. Strong leak through obscures the first $10 \mathrm{~km}$, but not the last $3 \mathrm{~km}$ of the return from the target. This $3-\mathrm{km}$ tail portion will be used for estimating the target.

To correctly partially decode the uncontaminated tail portion, the contaminated part must be eliminated; this is accomplished by replacing all of the samples from that portion of the receive signal with zeroes in the algorithm. Additional tapering is applied to have a smooth transition. Figure 3 illustrates this process. To generate the window function shown in Fig. 3, which will be multiplied with the returned signal from each pulse, it is necessary to start this function with zeroes, for as many samples as the transmit pulse, then the following samples will gradually ramp up from zero to one. This number of samples can be defined by the user as a small percentage of the pulse width. Finally, the remaining samples of the window function will be all ones.
Matched filter equation, shown in Eq. (2), is used to compress the received signal (Skolnik 2001a; Levanon and Mozeson 2004):

$$
y(n)=\sum_{\tau} x(\tau+n) x_{t}^{*}(\tau),
$$

where $y(n)$ is the compressed signal, $x(n)$ is the received signal, $x_{t}(n)$ is the matched filter (template), the asterisk $(*)$ is the complex conjugate operator, and $n$ corresponds to the sample index in the range. As explained before, the received signal is multiplied by a window function $w(n)$ in order to eliminate leakage from transmission. The new received signal is expressed in Eq. (3):

$$
x^{\prime}(n)=w(n) x(n)
$$

This modified received signal results in a different compressed signal $y^{\prime}(n)$, or partial decoding, which is expressed in Eq. (4):

$$
y^{\prime}(n)=\sum_{\tau} x^{\prime}(\tau+n) x_{t}^{*}(\tau)
$$

This new compressed received signal is called a progressive compressed signal. The leak through is eliminated. Because of using the residual tail of the received signals for compression, 


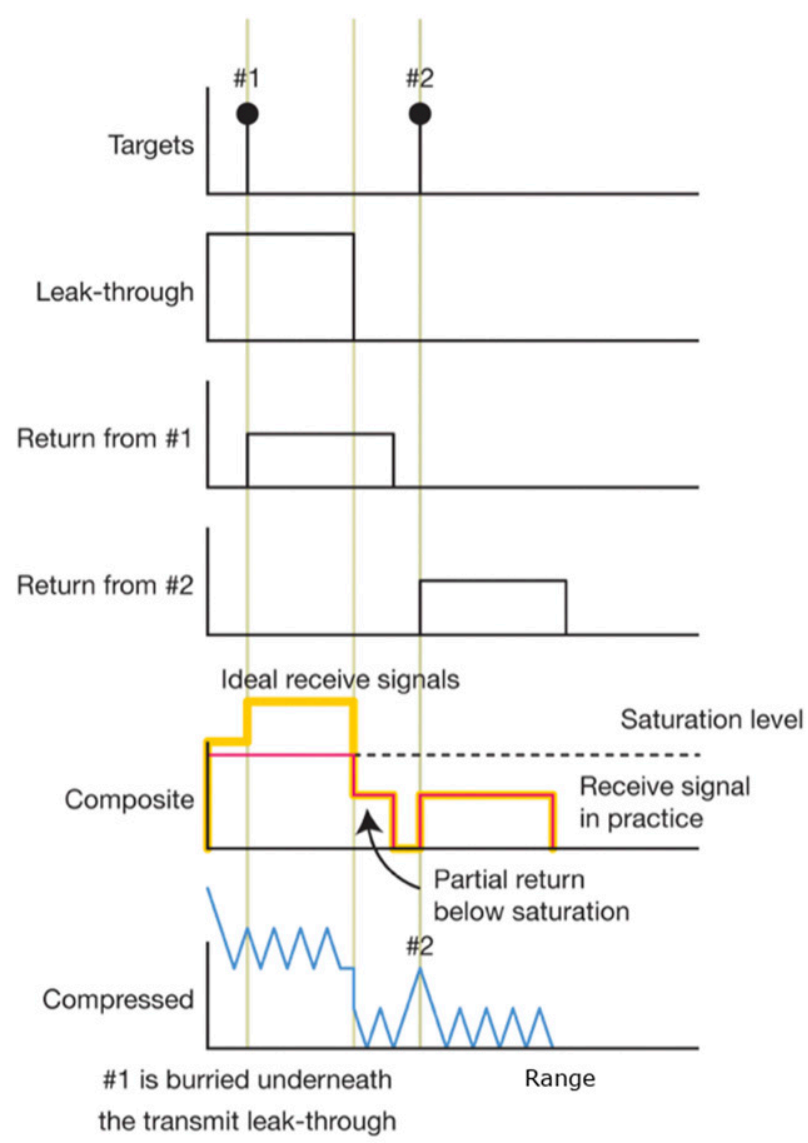

\section{Conventional Method}

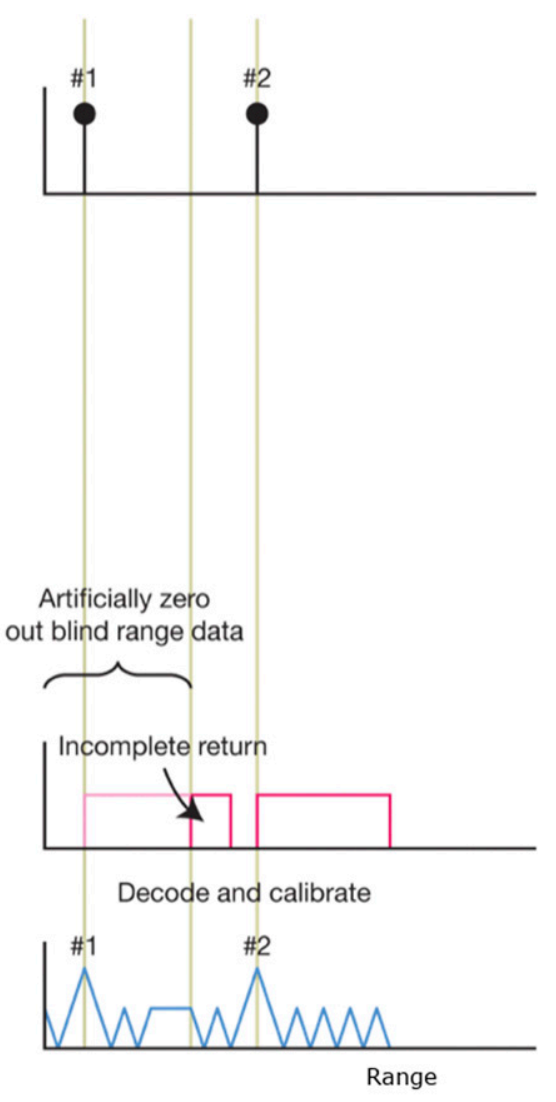

New Method

FIG. 2. The principle of partial decoding. (left) The target inside the blind range becomes obscured by strong leak through when processed using the conventional method (LPC). (right) When PPC is applied, the target can be correctly estimated, as is shown on the new method (PPC) plot.

the progressive compressed signal needs to be compensated differently for reflectivity estimation.

\section{2) CALibration}

As mentioned earlier, it is necessary to calculate a calibration or compensating factor to properly estimate the reflectivity values inside the blind range. Note that the tail uncontaminated portion is a function of range. For example, using a $10-\mathrm{km}$ transmit pulse, with two targets located at 5 and $8 \mathrm{~km}$, both of which are inside the blind range, the end of the return signals from each target will be at 15 and $18 \mathrm{~km}$, respectively. After the windowing process, the uncontaminated remaining

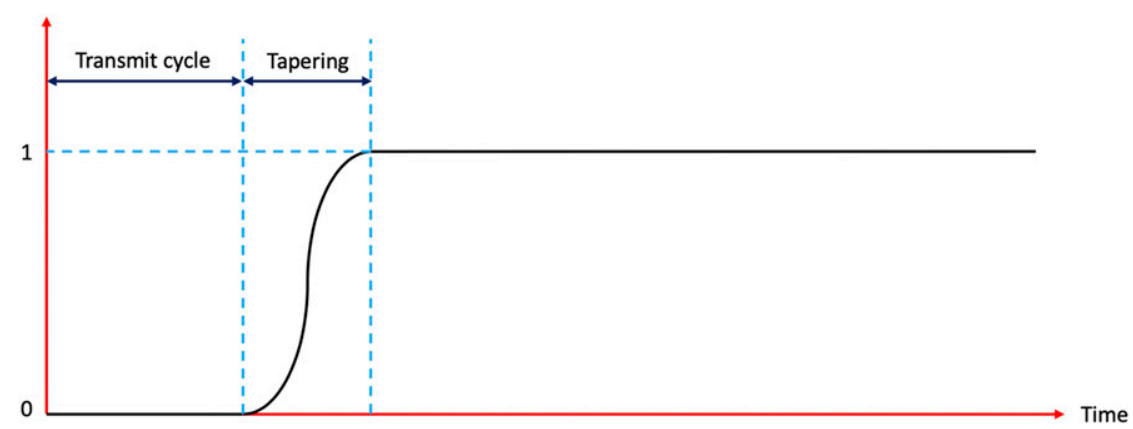

FIG. 3. Depiction of the zeroing-out process. An illustration of the window function used to eliminate leak through, where zeroes replace these samples with a gradual transition to one after leak through. 
portions are $5 \mathrm{~km}(10-15 \mathrm{~km})$ and $8 \mathrm{~km}(10-18 \mathrm{~km})$ from these two targets (pulse compression has only this many signals from each target). Therefore, after pulse compression, it is necessary to calibrate for the $50 \%$ and $80 \%$ signal availability of these two targets, respectively, for reflectivity estimation. The calculation of the calibration factor is described as follows.

Knowing the transmitted waveform, the window, and the position in range, a scaling factor can be calculated and used to calibrate the targets. Then, the target reflectivity at each range can be estimated. From Eq. (4), the compressed signal $y^{\prime}(n)$ contains just a portion of the received signal, and the required $y(n)$ is the compressed signal if there is no leak through and no blind range. It is not possible to obtain the $y(n)$ desired, but it can be estimated by multiplying a scaling factor, denoted as $s(n)$, to $y^{\prime}(n)$. The estimated compressed signal is named $y_{e}(n)$ :

$$
\begin{aligned}
& y_{e}(n)=s(n) y^{\prime}(n), \\
& y_{e}(n)=s(n) \sum_{\tau} x^{\prime}(\tau+n) x_{t}^{*}(\tau) .
\end{aligned}
$$

From Eqs. (5) and (6), the scaling factor $s(n)$ can be derived and is shown in Eq. (7):

$$
s(n)=\sqrt{\frac{\sum_{i}\left|x_{t}(i)\right|^{2}}{\sum_{i}\left|w(i) x_{t}(i)\right|^{2}}}
$$

Then, the new scaled and compressed output represents the estimated fully compressed received signal in the blind range free from leak through. This new scaled and compressed signal is used for radar product calculations (e.g., reflectivity factor, radial velocity), and represents the last step of this PPC algorithm. From here, the radar product calculation is done using standard implementations.

On the downside, there are some trade-offs of using just the tail portion to estimate the received signals inside blind range, such as a loss in range resolution, a shift in the mainlobe, and a decrease in sensitivity as the range is closer to the radar. In the following sections, a more in-depth performance analysis and the advantages and disadvantages of the PPC technique will be discussed.

\section{b. Sensitivity analysis}

One of the primary advantages of the proposed technique over other methods (e.g., George et al. 2010; Bharadwaj and Chandrasekar 2012; Cheong et al. 2013) is the sensitivity gain inside the blind range. To define sensitivity, radar reflectivity factor $(Z)$ will first be defined. Equation (8) defines $Z$ (Doviak and Zrnić 2006c):

$$
Z=\frac{P_{r} 2^{10} \ln (2) \lambda^{2} r_{0}^{2} l_{r}^{2} l_{r}}{\pi^{3} P_{t} g^{2} g_{s} \theta^{2} c \tau\left|K_{w}\right|^{2}}
$$

where $P_{r}$ is the power returned to the $\operatorname{radar}(\mathrm{W}), \lambda$ is the wavelength $(\mathrm{m}), r_{0}$ is the range from the radar to the target $(\mathrm{m})$, $l$ and $l_{r}$ are the loss factors (unitless), $P_{t}$ is the peak power transmitted (W) from the radar, $g$ and $g_{s}$ are gain factors (unitless), $\theta$ is the beamwidth (rad), $c$ is the speed of light $\left(\mathrm{m} \mathrm{s}^{-1}\right), \tau$ is the pulse width (s), and $K_{w}$ is the complex dieletric factor of water (unitless).

Radar sensitivity is the minimum reflectivity factor $\left(Z_{\min }\right)$ at a given range $r$ that corresponds to the minimum detectable radar signal. A common conception is that the lower the value of $Z_{\min }$ achievable, the better the sensitivity of the radar. To obtain this $Z_{\min }$, it is assumed that the received power is about the same as the power from the noise floor, which for PX-1000 is about $-110 \mathrm{dBm}$. Then, in order to mathematically explain the sensitivity values, the radar reflectivity factor in Eq. (8) is used, assuming the received power $P_{r}$ is the same as the noise floor, and $Z$ is the sensitivity value $Z_{\min }$.

The value of $Z_{\min }$ changes as a function of $\tau: Z_{\min }$ is inversely proportional to $\tau$, so the larger $\tau$ gets, the lower $Z_{\text {min }}$ becomes, increasing the sensitivity of the radar. Some techniques to mitigate the blind range use different $\tau$ values for the visible and blind ranges (e.g., George et al. 2010; Bharadwaj and Chandrasekar 2012; Cheong et al. 2013), since they transmit long and fill pulses. As a consequence, there is jump in radar sensitivity between the blind and visible ranges.

When PPC is implemented, the situation becomes slightly more complex, especially inside the blind range. Even though $\tau$ is constant during transmit, inside the blind range during receive, however, it is a function of range $\tau(r)$. This is caused by the windowing process, which effectively reduces the length of $\tau$ that is left for each range bin (remaining tail portion). The effective pulse width $\tau(r)$ starts at zero, linearly becomes larger as the range increases away from the radar and, eventually, $\tau(r)=\tau$ beyond the blind range $\left(r \geq R_{b}\right)$.

Then, for PPC, $Z_{\min }$ is now also inversely proportional to $\tau(r)$, and it gradually increases as the range gets closer to the radar:

$$
Z_{\text {min }}=\frac{P_{r}(r) 2^{10} \ln (2) \lambda^{2} r_{0}^{2} l^{2} l_{r}}{\pi^{3} P_{t} g^{2} g_{s} \theta^{2} c \tau(r)\left|K_{w}\right|^{2}} .
$$

To illustrate this phenomenon, a software routine to compute radar sensitivity according to Eq. (9) for different radar parameters and blind range mitigation techniques has been developed.

The PX-1000 has a 200-W transmitter on each polarization and its radar parameters $\left(P_{r}, P_{t}, \lambda\right.$, etc. $)$ and a $67-\mu$ s optimized frequency modulation (OFM) waveform (Kurdzo et al. 2014; Kurdzo 2015) are used as the baseline. For the OFM waveform, its baseband representation, frequency function, and ambiguity function are shown in Fig. 4. The radar sensitivity profiles using the time-frequency multiplexing (TFM), LPC, and PPC techniques are shown in Fig. 5. The TFM radar sensitivity is indicated by the yellow line. The abrupt change between the blind range and visible range is obvious, since they use different waveforms with pulse widths of 2 and $67 \mu$ s, respectively. The PPC sensitivity curve with a $67-\mu$ s pulse width, shown in red, has a continuous transition from the blind range to the visible range. The green line is from a radar using a $5-\mathrm{kW}$ transmitter and a $0.5-\mu$ s pulse width, similar to the magnetronbased Collaborative Adaptive Sensing of the Atmosphere (CASA) Integrated Project 1 (IP1) (Junyent et al. 2010). 

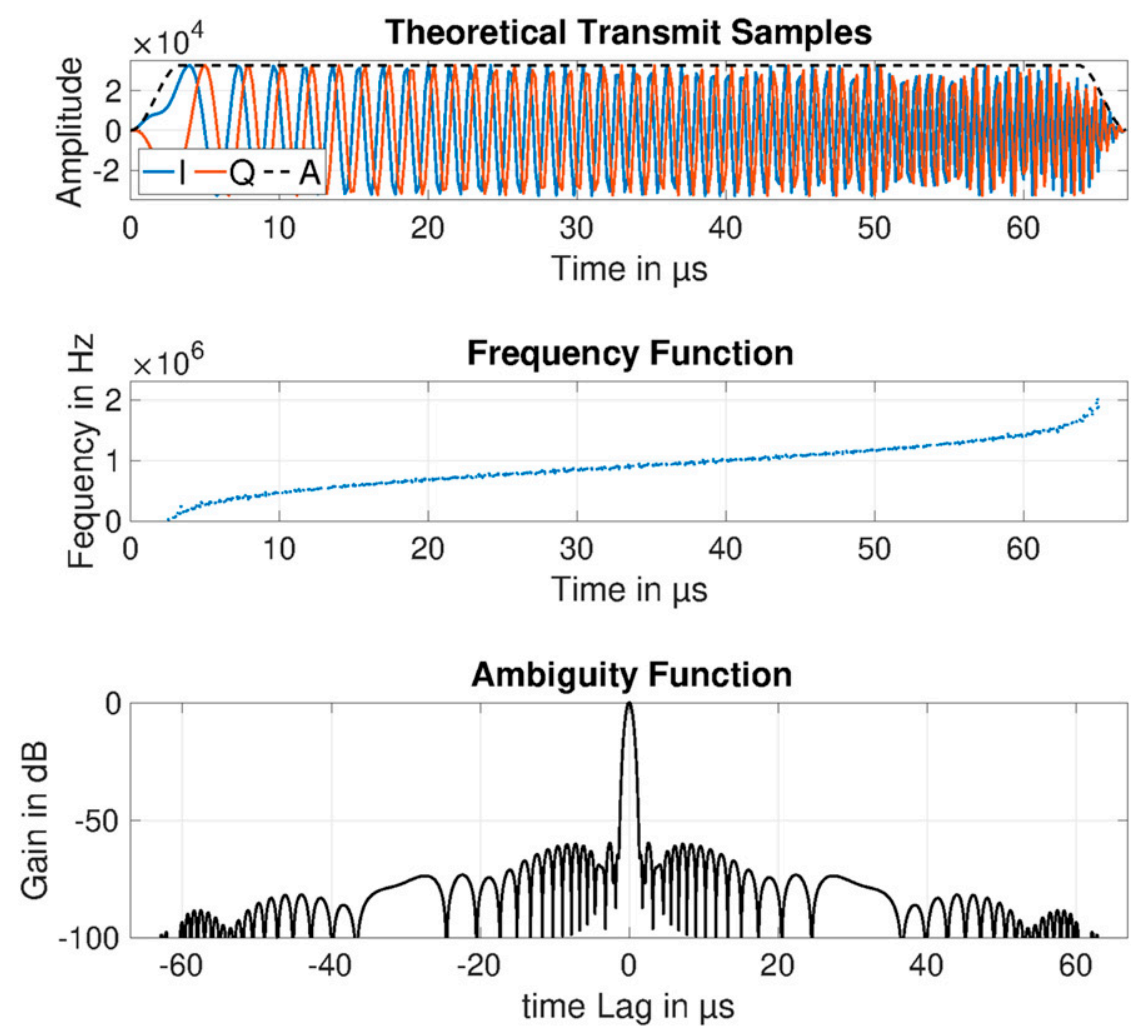

FIG. 4. Waveform used for PPC experiments. An NLFM waveform has been selected, designed, and optimized for the PX-1000 (Kurdzo et al. 2014; Kurdzo 2015). Only the long pulse is used since the PPC technique requires no fill pulse.

The black dashed line indicates a hypothetical radar sensitivity of a $67-\mu$ s pulse configuration with no blind range.

The PPC technique provides comparable sensitivity values to the CASA-like radar system with a $5-\mathrm{kW}$ transmitter at a $0.5-\mu \mathrm{s}$ pulse width. The sensitivity profile obtained using the PPC technique has a more gradual slope inside the blind range. It is noted here that the PPC technique still has a slightly poorer performance in sensitivity at close range, compared to the $5-\mathrm{kW}$ system.

\section{c. Range resolution}

Pulse compression increases range resolution and radar sensitivity. The range resolution can be attributed to the effective bandwidth of the waveform. Due to the zero-out process, the residual partial waveform retains only a portion of the bandwidth and results in a loss of resolution. In this section, the performance of PPC in terms of range resolution will be examined.

Figure 4 shows the transmitted waveform developed for the PX-1000 (Kurdzo et al. 2014; Kurdzo 2015). Specifically, the long pulse, which belongs to an NLFM waveform family, is shown.

Figure 6 (top) shows the impulse response of a few point targets and illustrates the loss of resolution as the range decreases. The loss of resolution can be attributed to the reduction of effective bandwidth as the portion of the waveform for compression is reduced as the range decreases. The bandwidth of the waveform is $2.2 \mathrm{MHz}$ and the pulse width is $67 \mu \mathrm{s}$.

The middle plot of Fig. 6 shows a function that demonstrates the shift of the mainlobe in range. This shift of the mainlobe is a drawback of the PPC technique. It is hypothesized that this effect is due to the alteration of the modulation that is caused by the zero-out process. This effect merits an in-depth analysis and it is worthwhile for a future work.

The bottom plot of Fig. 6 shows the loss of range resolution as targets get closer to the radar. This loss is specific to PPC and it is a consequence of the partial decoding process. In the partial decoding process, parts of the received signal are zeroed out, using only the uncontaminated tail portion. This tail portion can be understood as a new pulse and came from the original pulse, which was frequency modulated with a specific bandwidth to guarantee a good radar resolution; however, the uncontaminated remaining tail portion only has a fraction of such bandwidth, which changes as a function of range.

As mentioned before, range resolution is proportional to the waveform bandwidth; that is, lower bandwidth results in lower range resolution. If a radar transmits an LFM waveform, the frequency and bandwidth are linearly distributed throughout the pulse: a $10 \%$ leftover tail means a $10 \%$ leftover bandwidth, which leads to $10 \%$ of the original resolution. However, if a radar transmits an NLFM waveform, like the one in the simulation and the PX-1000 system, the frequency is nonlinearly 


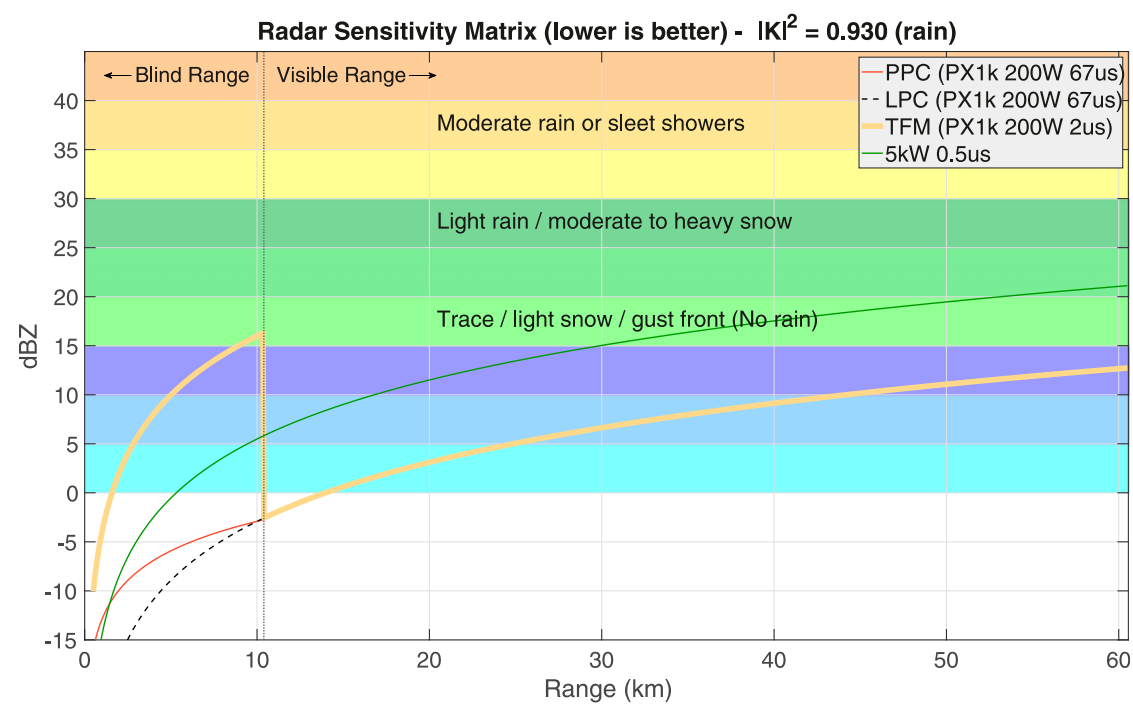

FIG. 5. Radar sensitivity of different systems. PPC provides a smooth, imperceptible transition in sensitivity between the blind and visible ranges. In addition, PPC provides better sensitivity values inside the blind range compared to TFM and has comparable sensitivity values to radar systems using higher-power transmitters.

distributed and, thus, the resolution behavior is more complex but is evaluated numerical by computing its ambiguity function.

In the same way that the mainlobes on the targets inside the blind range are affected due to PPC, which was explained in detail previously, the range sidelobes for those targets are affected as well. Figure 7 presents the ambiguity function for simulated progressive pulse-compressed targets that are located at different range gates inside the blind range, but now with a larger gain axis range. From Fig. 7 , it can be seen that the sidelobes are not symmetrical for the targets inside the blind range, and that some range sidelobes for close targets are extremely small toward the radar and larger away from the radar. This effect, which is more pronounced for close ranges, will be a topic of future study.

\section{Results}

\section{a. Simulation results}

A simulation was undertaken to study the potential realistic performance of a complex transmit waveform. The waveform
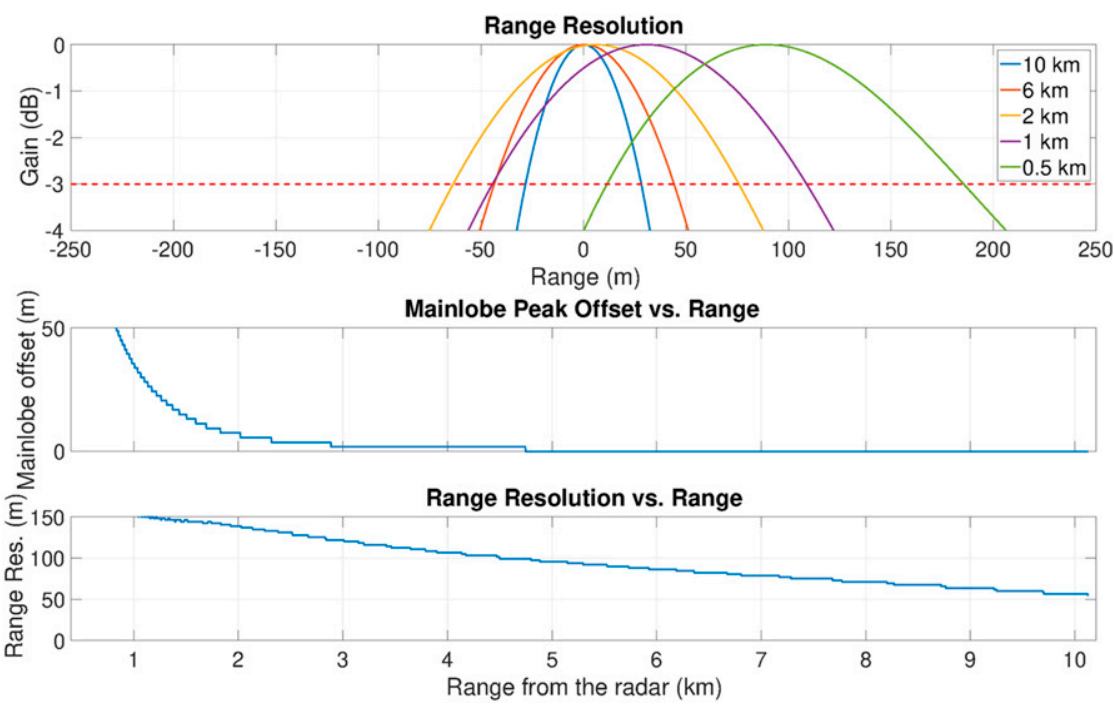

FIG. 6. Various waveform behaviors of the PPC technique. (top) The waveform ambiguity functions at various ranges, where the loss of range resolution from closer targets is easy to see. In addition, there is also a range shift of the peaks. (middle),(bottom) The shifts of the peaks and range resolution, respectively, as a function of range. 


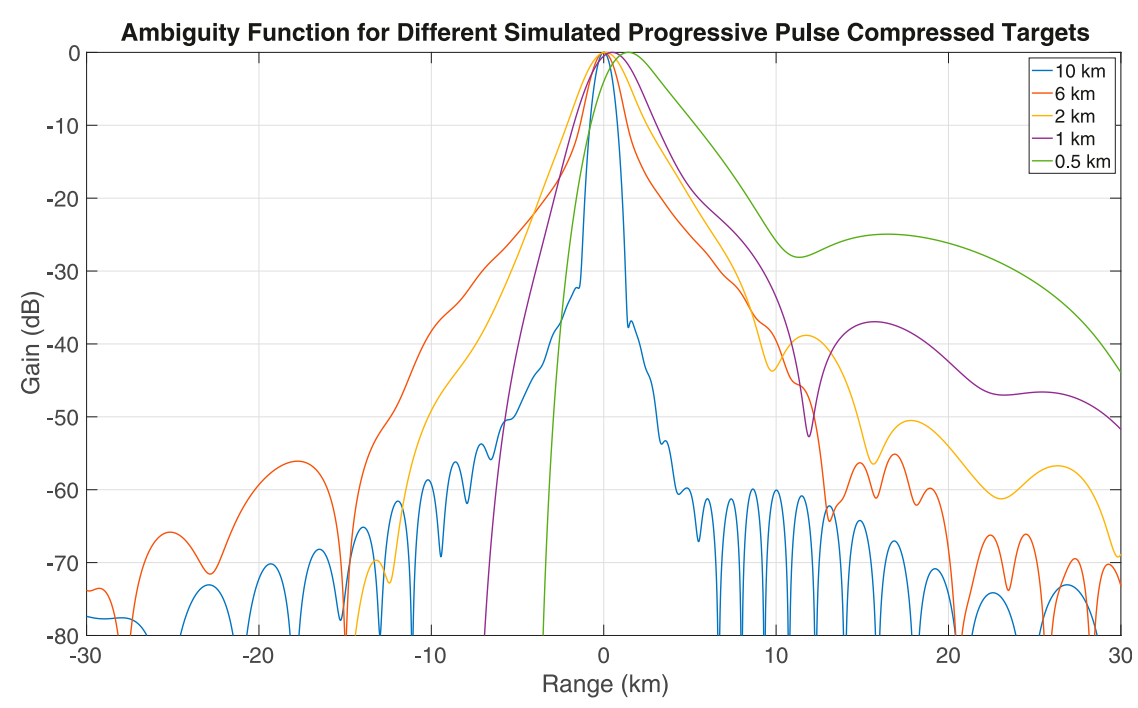

FIG. 7. As in Fig. 6, the ambiguity function is provided for simulated progressive pulse compressed targets located at different range gates, but with a larger gain axis. As it can be seen, not only the mainlobe is affected by the range of the target, but also the range sidelobes.

used is shown in Fig. 4; this waveform is identical to the long pulse of the one presented by Kurdzo et al. (2014) and Kurdzo (2015) and identical to the long pulse currently transmitted by the PX-1000.

Four point targets are simulated. Two are located inside the blind range with the remaining outside the blind range. The leak through is simply a power-altered and phased-shifted copy of the transmitted waveform, similar to the leakage formed in the PX-1000. Realistic noise level was also added.

Figure 8 shows the simulation setup and return-power profile from the LPC and the PPC techniques. The top panel shows the target locations. When the received signal is processed using the LPC, as shown in the middle panel, two targets are totally obscured. The bottom panel shows results processed using the PPC, and the two previously obscured targets are now visible.

To compare the results between the PPC and LPC, the subplots are divided into two regions, the visible range and the blind range. In the visible range the results from both LPC and PPC are identical, targets show the same peak power and the same resolution, and the noise level is also the same. Recall that the PPC technique eliminates the uncontaminated signal using a window function to zero out the returned signal in the blind range, which only affects the echoes from targets inside
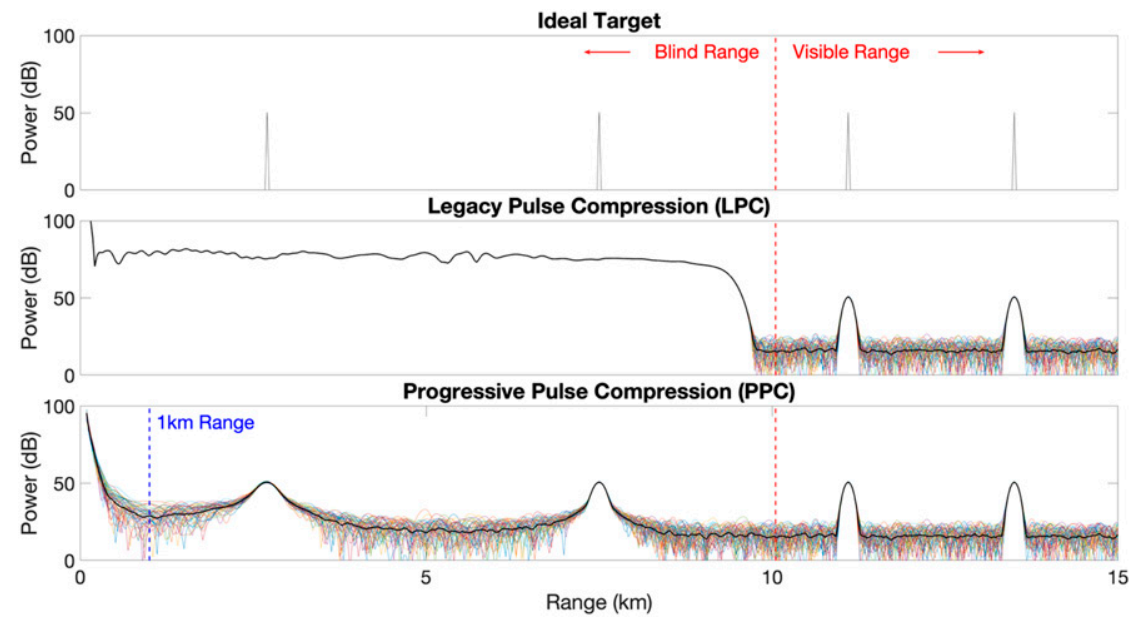

FIG. 8. Simulation setup and results. (top) Target locations. (middle) Simulation results from the LPC. (bottom) Simulation results from the PPC. The simulation demonstrates that when processed using the LPC, the targets inside the blind range are obscured by the leak through. By contrast, when using the PPC over the same samples, the targets are visible. 
the blind range. The pulse width of this simulation is $10 \mathrm{~km}$, which means that the blind range is also $10 \mathrm{~km}$. The target at $11 \mathrm{~km}$ produces a received echo that spans the range in between 11 and $21 \mathrm{~km}$. Consequently, the contamination does not affect this target and the windowing process has no effects on this target. So, partial decoding at this range is exactly the same as the LPC and TFM and every range gate outside the blind range. This is why LPC and PPC have the same results and performance outside the blind range.

On the other hand, inside the blind range, LPC suffers from strong leak through, which obscures everything. In contrast, PPC can correctly estimate the two targets. The loss in range resolution is apparent. As explained earlier, range resolution is lower closer to the radar as the leftover tail portion is shorter. This effect is manifested into wider targets being produced closer to the radar. In addition, the mainlobe peak is slightly shifted. With a proper PPC calibration, the correct reflectivity can be estimated as expected.

\section{b. Experimental results: PPC versus TFM}

The PPC technique has been implemented on the PX-1000 radar system. Figures 9 and 10 show radar products calculated using the PX-1000 data. The radar products SNR, reflectivity factor, radial velocity (presented in Fig. 9), and differential reflectivity, differential phase (PhiDP), and correlation coefficient (RhoHV) (presented in Fig. 10) were processed using TFM and PPC from a dataset gathered at 2233 UTC 14 September 2014. The dataset used to process results in Figs. 9 and 10 was chosen because it is an example that clearly shows the improvements in radar sensitivity of the PPC technique over the TFM, thanks to the presence of weather inside the blind range.

In the visible range (i.e., outside the blind range), results from both PPC and TFM are identical as expected. Both techniques share the same input data and matched filter. The real-time software of the $\mathrm{PX}-1000$ has been updated to use the PPC and no longer transmit the fill pulse of the TFM. (The realdata imagery is available through a web portal at https:// arrc.ou.edu/px1000/).

Without using a fill pulse like the TFM method, there is no abrupt change in sensitivity between the blind range and the visible range, which is noticeable in the TFM reflectivity factor subplot in Fig. 9. This jump in sensitivity impacts all radar products. The PPC has a continuous transition in sensitivity between the blind range and visible range. The loss in sensitivity is gradual but the loss in range resolution is more noticeable closer to the radar. Because of the sensitivity improvement, more weather signals can be observed inside the blind range, especially at the northern portion of the PPI plot, approximately $8-\mathrm{km}$ range. Besides the reflectivity, all other radar products also exhibit similar sensitivity improvement and spatial continuity.

Figure 11 shows the minimum detectable reflectivity factor $\left(Z_{\text {min }}\right)$ values from the TFM and the PPC techniques as a function of range from the dataset gathered at 2233 UTC 14 September 2014. Minimum $Z$ value corresponds to the minimum radar reflectivity factor from all radials, in every sample point in range. This is one way to estimate the radar sensitivity. Experimental results from the estimated radar sensitivity values overlap almost perfectly with the sensitivity values obtained from simulation. This indicates that sensitivity is indeed higher inside the blind range using the PPC technique compared to the TFM technique. This can be attributed to the increase in available (leftover) pulse width $\tau(r)$ as $r$ increases, indicated by Eq. (9). Compared to the TFM technique, which uses a fill pulse of a fixed width of $2 \mu \mathrm{s}$, the radar sensitivity of the PPC technique is higher where $r \geq 2 \mathrm{~km}$.

\section{Discussion and conclusions}

Over the years, different radar technologies have been developed to overcome different hardware limitations. Solidstate radars need to transmit a long pulse and use pulse compression to overcome the low peak power of solid-state transmitters and achieve the desired radar sensitivity and range resolution. It has been commonly recognized that a negative effect of using long pulse is the blind range, which is a result of leak through overpowering the signals of interest. The blind range is directly proportional to the pulse width. For example, a $67-\mu$ s results in a $10-\mathrm{km}$ blind range, which means that the first $10 \mathrm{~km}$ of data are obscured.

In the literature, different implementations have been proposed to solve the bind range limitation (e.g., George et al. 2010; Bharadwaj and Chandrasekar 2012; Cheong et al. 2013) and they all share a common method of using additional pulses, referred to as the fill pulses, that are transmitted successively. The use of fill pulses, usually shorter and have different frequencies, has a few drawbacks. It requires more computational time to process for those fill pulses. The system also requires more bandwidth to accommodate the fill pulses at different frequencies, which is an expensive resource. More importantly, since the fill pulses are generally shorter and are used to process for data inside the blind range, the achievable sensitivity is lower, and inadvertently introduced an abrupt change (or multiple abrupt changes from multiple fill pulses) in sensitivity across the transition range.

On the other hand, the PPC takes advantage of the long pulse, which produces long echoes. Thanks to that, even the leak through from the transmission only obscures a portion of the received signals. In general, the received echoes inside the blind range are not completely obscured. An uncontaminated portion is still available and can be used to correctly estimate the target reflectivity.

The PPC technique can be divided into three steps. The first step is the elimination of the leak through by multiplying the raw received signal by a window function that gradually ramps from zero to one near the visible range, the second step is same as conventional pulse compression, and the third step is a new reflectivity correction that compensates for the partial returned signals. The combination of first and second steps is an equivalence of using different matched filters (templates) at different range in pulse compression to extract the uncontaminated echoes. A windowing process is simpler and allows for the subsequent pulse compression process to use the existing implementation. The PPC technique requires no 


\section{TFM}

\section{SNR}

a)

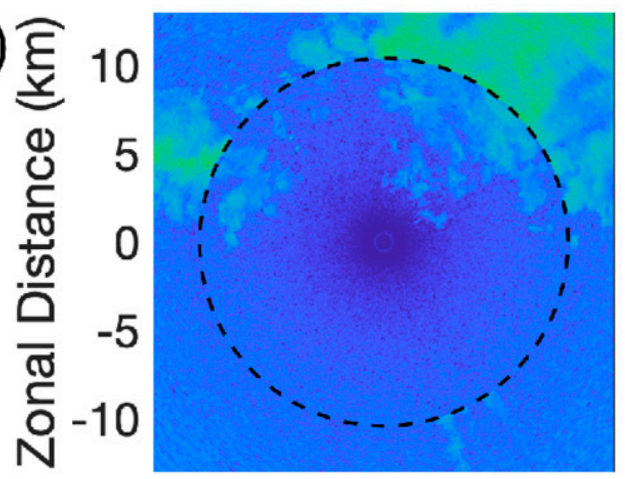

PPC

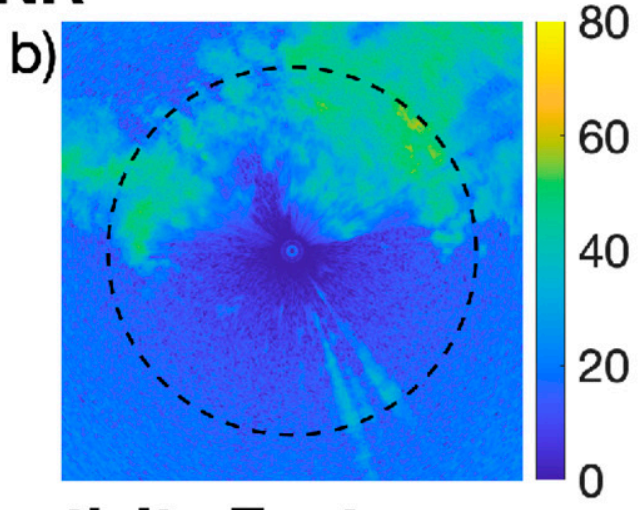

\section{Radar Reflectivity Factor}

c)

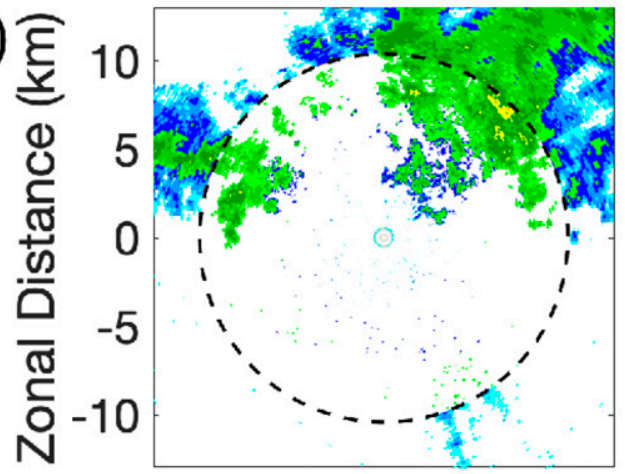

d)

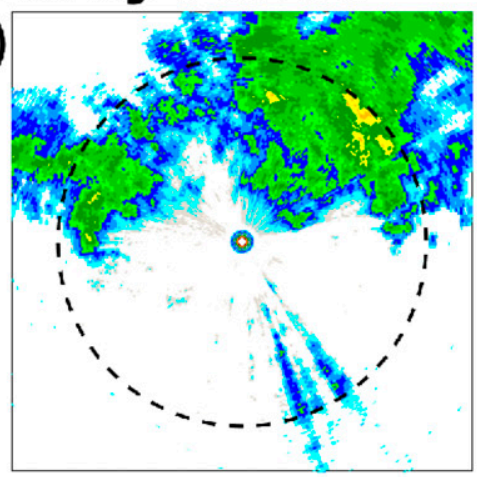

80

\section{Radial Velocity}

e)

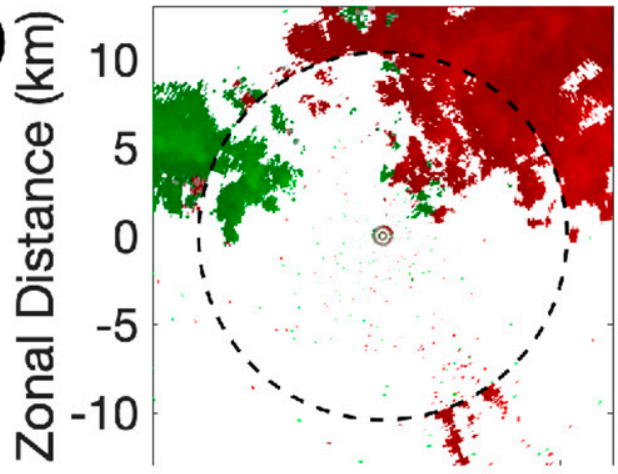

$-10$

10 f)

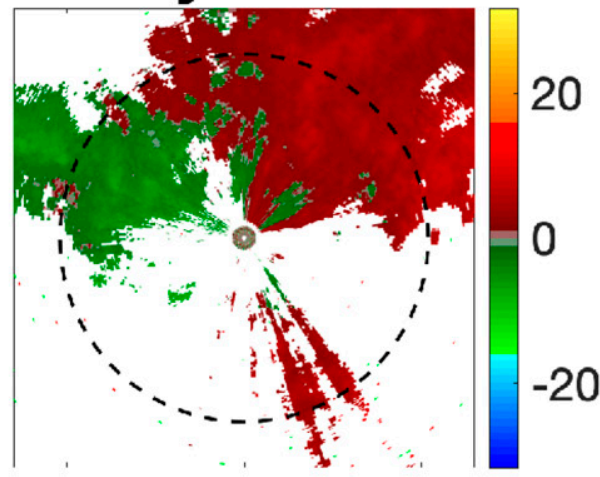

$-10$
0

\section{Meridional Distance $(\mathrm{km})$ Meridional Distance $(\mathrm{km})$}

FIG. 9. (left) Products processed using TFM: (a) SNR, (c) reflectivity factor, and (e) radial velocity. (right) Products processed using PPC: (b) SNR, (d) reflectivity factor, and (f) radial velocity. In these plots it is shown that radar sensitivity is higher inside the blind range on PPC and that the discontinuity in sensitivity has been eliminated. Consequently, PPC results show more information inside the blind range than TFM results.

hardware modification and, thus, can be applied to many systems as a software update.

The main advantage of the PPC techniques is an increase in the radar sensitivity inside the (old) blind range. Radar sensitivity, as explained before, is proportional to the pulse width $\tau$. Different from using fill pulses, the PPC uses the uncontaminated tail portion of the returned pulse. Therefore, the pulse width $\tau$ of the (new) uncontaminated received pulse gradually increases as the 

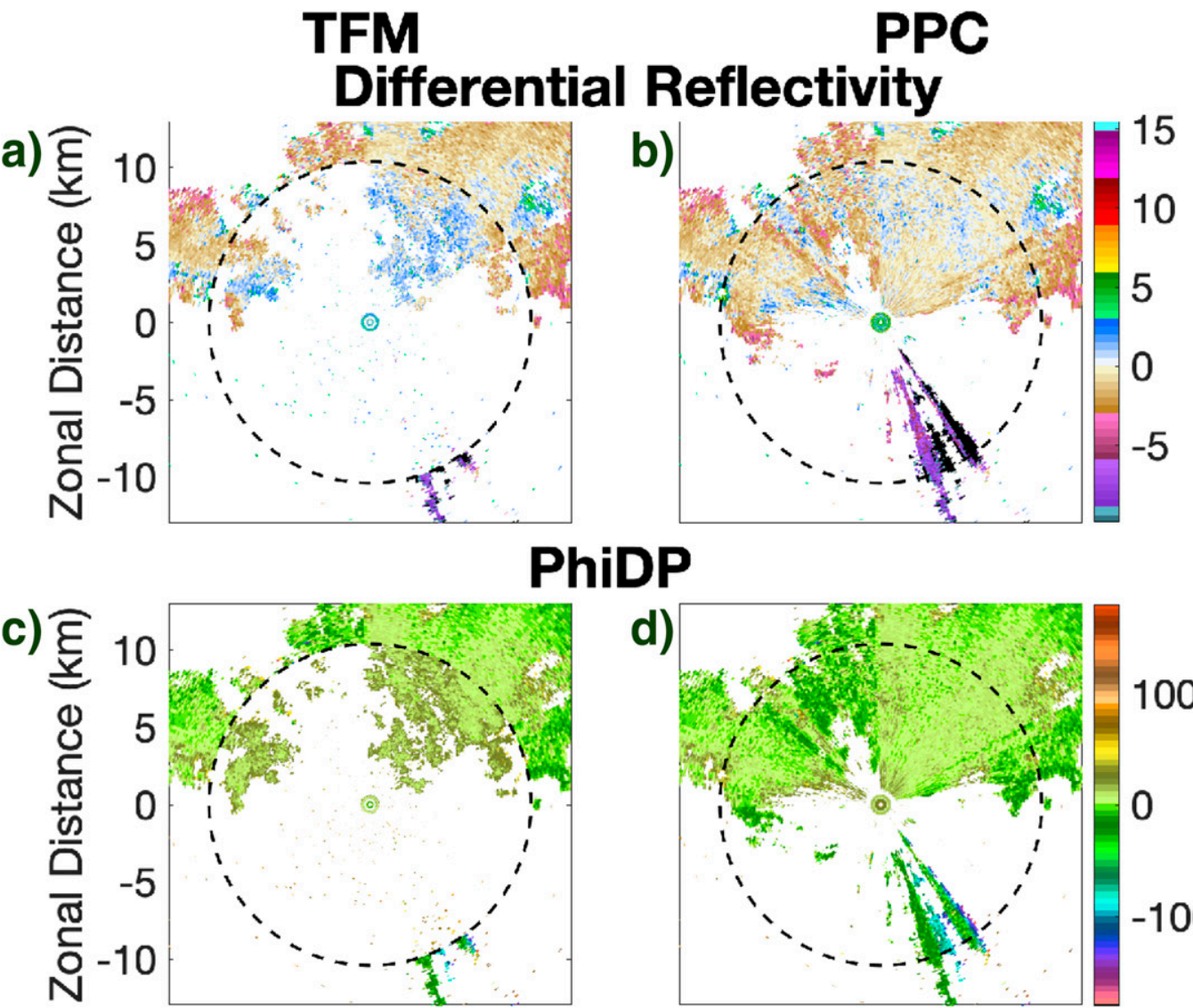

d)

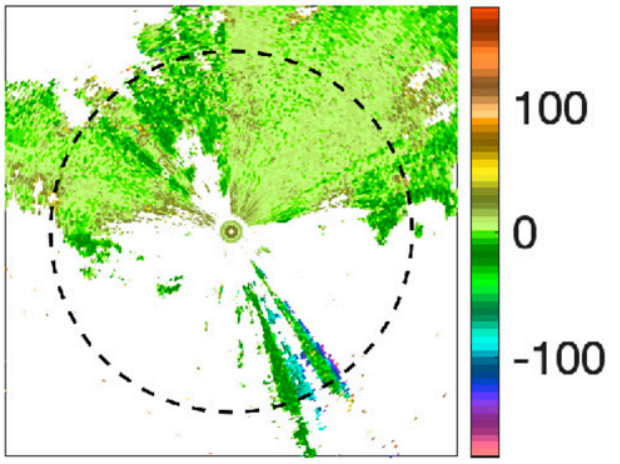

RhoHV

e)

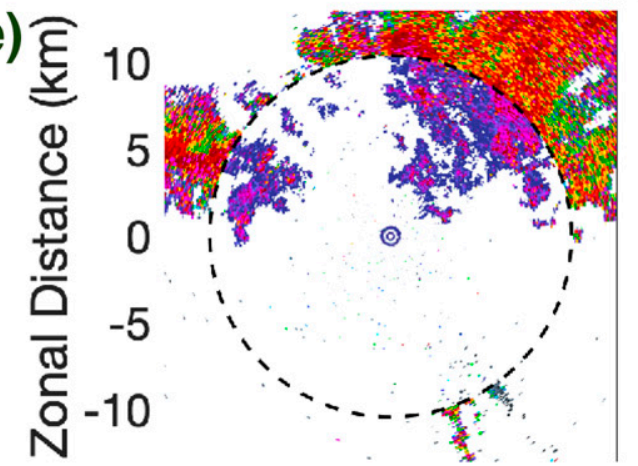

$-10$

0

10 f)

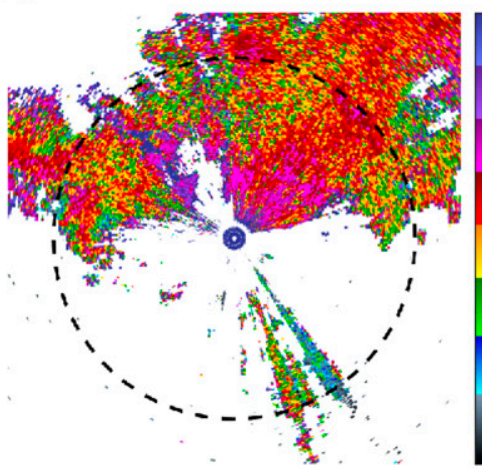

1.05

1.02

0.99

0.96

0.93

0.83

0.73

0

10

\section{Meridional Distance $(\mathrm{km})$ Meridional Distance $(\mathrm{km})$}

FIG. 10. (left) Products processed using TFM: (a) Differential reflectivity, (b) PhiDP, and (c) RhoHV. (right) Products processed using PPC: (d) Differential reflectivity, (e) PhiDP, and (f) RhoHV. In these plots it is shown that radar sensitivity is higher inside the blind range on PPC and that the discontinuity in sensitivity has been eliminated. Consequently, PPC results show more information inside the blind range than TFM results.

range $r$ increases and eventually a full pulse is received outside of the blind range; that is, everything remains the same in the visible range.

On the downside, since the PPC technique uses the uncontaminated tail portion of the received signal, there is a loss of range resolution and shift of compressed mainlobe. The loss of resolution can be attributed to the tail portion that contains only a portion of the bandwidth. This effect was numerically quantified and presented in this paper. The shift of compressed mainlobe is simply a shift of the maximum gain when an 

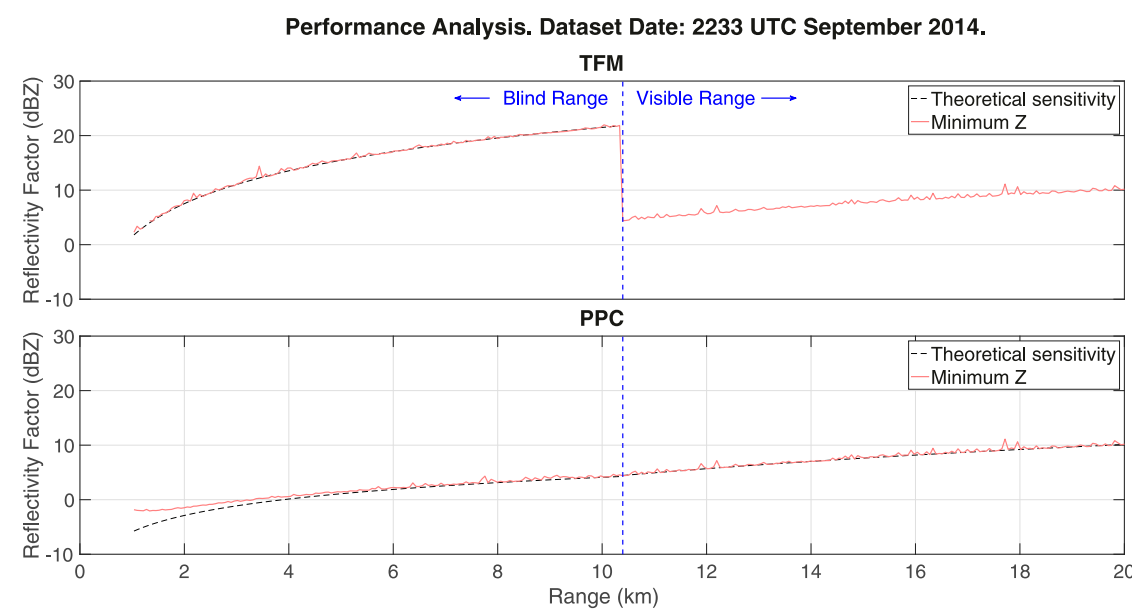

FIG. 11. Estimated radar sensitivity using the (top) TFM and (bottom) PPC techniques from the dataset gathered in 2233 UTC 14 Sep 2014. They are obtained by finding the minimum detectable reflectivity factor values from a collection of radials.

incomplete echo is processed with the matched filter. Future work includes a more in-depth study and mitigation of such artifact.

Another potential disadvantage of the PPC technique is its effect on the range sidelobes for the targets inside the blind range. Sidelobes for those targets are not symmetrical and those toward the radar can be extremely small (and larger away from the radar). Furthermore, for those targets, the sidelobe levels worsen as the target gets closer to the radar. Future work includes a more in-depth study of any impacts and the need for a mitigation strategy.

The PPC technique represents a new leap in blind range mitigation and its implementation is simple. It requires no hardware modifications and all the necessary changes are within the receive portion the digital signal processor. There is no changes to the transmit portion of the software. Currently, the PPC technique has been implemented to the PX-1000 system (a real-time web portal is available at https://arrc.ou.edu/px1000).

Acknowledgments. This work was supported by the NOAA National Severe Storms Laboratory through Cooperative Agreement NA15OAR4320115.

Data availability statement. PX-1000 data are available from the authors upon request.

\section{REFERENCES}

Balajti, I., G. Kende, and E. Sinner, 2012: Increased importance of VHF radar in ground-based air defense. IEEE Aerosp. Electron. Syst. Mag., 27, 4-18, https://doi.org/10.1109/ MAES.2012.6145436.

Bharadwaj, N., and V. Chandrasekar, 2012: Wideband waveform design principles for solid-state weather radars. J. Atmos. Oceanic Technol., 29, 14-31, https://doi.org/10.1175/JTECHD-11-00030.1.

Cheong, B. L., K. Redmond, R. D. Palmer, Y. Zhang, M. Yeary, and T.-Y. Yu, 2013: PX-1000: A solid-state polarimetric $\mathrm{X}$-band weather radar and time-frequency multiplexed waveform for blind range mitigation. IEEE Trans. Instrum. Meas., 62, 3064-3072, https://doi.org/ 10.1109/TIM.2013.2270046.

De Witte, E., and H. D. Griffiths, 2004: Improved ultra-low range sidelobe pulse compression waveform design. IEEE Electron. Lett., 40, 1448-1450, https://doi.org/10.1049/el:20046548.

Doviak, R. J., and D. S. Zrnić, 2006a: Considerations in the observation of weather. Doppler Radar and Weather Observations, 2nd ed. Dover Publications Inc., 160-208.

—, and — 2006b: Doppler Radar and Weather Observations. 2nd ed. Dover Publications Inc., 562 pp.

- , and - 2006c: Weather signals. Doppler Radar and Weather Observations, 2nd ed. Dover Publications Inc., 64-86.

George, J., N. Bharadwaj, and V. Chandrasekar, 2008: Considerations in pulse compression design for weather radars. IGARSS 2008-2008 IEEE Int. Geoscience and Remote Sensing Symp., Vol. 5, Boston, MA, IEEE, V-109-V-112, https:// doi.org/10.1109/IGARSS.2008.4780039.

—_, K. V. Mishra, C. M. Nguyen, and V. Chandrasekar, 2010: Implementation of blind zone and range-velocity ambiguity mitigation for solid-state weather radar. 2010 IEEE Radar Conf., Arlington, VA, IEEE, 1434-1438, https:// doi.org/10.1109/RADAR.2010.5494392.

Hysell, D., 2018a: Introduction. Antennas and Radar for Environmental Scientist and Engineers, Cambridge University Press, 1-28.

_ 2018b: Pulse compression. Antennas and Radar for Environmental Scientist and Engineers, Cambridge University Press, 195-217.

Junyent, F., V. Chandrasekar, D. McLaughlin, E. Insanic, and N. Bharadwaj, 2010: The CASA integrated project 1 networked radar system. J. Atmos. Oceanic Technol., 27, 61-78, https://doi.org/10.1175/2009JTECHA1296.1.

Keeler, R. J., and C. A. Hwang, 1995: Pulse compression for weather radar. Proc. Int. Radar Conf., Alexandria, VA, IEEE, 529-535, https://doi.org/10.1109/RADAR.1995.522603.

Kurdzo, J. M., 2015: Pulse compression waveforms and applications for weather radar. Ph.D. thesis, University of Oklahoma, 316 pp.

—, B. L. Cheong, R. D. Palmer, G. Zhang, and J. B. Meier, 2014: A pulse compression waveform for improved-sensitivity weather radar observations. J. Atmos. Oceanic Technol., 31, 2713-2731, https://doi.org/10.1175/JTECH-D-13-00021.1. 
Levanon, N., and E. Mozeson, 2004: Matched filter. Radar Signals, 1st ed. John Wiley and Sons Inc., 20-33.

Mudukutore, A. S., V. Chandrasekar, and R. J. Keeler, 1998: Pulse compression for weather radars. IEEE Trans. Geosci. Remote Sens., 36, 125-142, https://doi.org/10.1109/ 36.655323 .

Nguyen, C. M., and V. Chandrasekar, 2014: Sensitivity enhancement system for pulse compression weather radar. J. Atmos. Oceanic Technol., 31, 2732-2748, https://doi.org/10.1175/JTECH-D-1400049.1.

O'Hora, F., and J. Bech, 2007: Improving weather radar observations using pulse-compression techniques. Meteor. Appl., 14, 389-401, https://doi.org/10.1002/met.38.

Pang, C., P. Hoogeboom, F. Le Chevalier, H. W. J. Russchenberg, J. Dong, T. Wang, and X. Wang, 2015: A pulse compression waveform for weather radars with solid-state transmitters. IEEE Geosci. Remote Sens. Lett., 12, 2026-2030, https:// doi.org/10.1109/LGRS.2015.2443551.
Pralon, L., B. Pompeo, G. Beltrao, H. Cioqueta, B. Cosenza, and J. Moreira, 2012: On a blind zone elimination method based on partial compression filter design using random waveforms for monostatic pulsed radars. IET Int. Conf. on Radar Systems (Radar 2012), Glasgow, United Kingdom, Institution of Engineering and Technology, C7.1, https://doi.org/10.1049/ cp.2012.1653.

Skolnik, M. I., 2001a: Detection of signals in noise. Introduction to Radar Systems, 3rd ed. McGraw-Hill Higher Education, 276-312.

, 2001b: Information for radar signals. Introduction to Radar Systems, 3rd ed. McGraw-Hill Higher Education, 313-402.

Torres, S. M., C. D. Curtis, and D. Schvartzman, 2017: Requirementdriven design of pulse compression waveforms for weather radars. J. Atmos. Oceanic Technol., 34, 1351-1369, https://doi.org/ 10.1175/JTECH-D-16-0231.1.

Wada, M., J. Horikomi, and F. Mizutani, 2009: Development of solid-state weather radar. 2009 IEEE Radar Conf., Pasadena, CA, IEEE, 1-4, https://doi.org/10.1109/RADAR.2009.4977071. 